\title{
Role of Molecular Architecture on Ion Transport in Poly(ethylene oxide)-based Polymer Electrolytes
}

\section{Supporting Information}

\section{Authors}

Chuting Deng ${ }^{1}$, Michael A. Webb ${ }^{4}$, Peter Bennington ${ }^{1}$, Daniel Sharon ${ }^{1,2,3}$, Paul F. Nealey ${ }^{1,2,3}$, Shrayesh N. Patel ${ }^{1,2}$, Juan J. de Pablo ${ }^{1,2,3 *}$

\section{Affiliations}

${ }^{1}$ Pritzker School of Molecular Engineering, University of Chicago, 5640 S Ellis Ave, Chicago, IL 60637, United States

${ }^{2}$ Center for Molecular Engineering, Argonne National Laboratory, 9700 South Cass Avenue, Lemont, IL 60439, United States

${ }^{3}$ Materials Science Division, Argonne National Laboratory, 9700 South Cass Avenue, Lemont, IL 60439, United States

${ }^{4}$ Department of Chemical and Biological Engineering, Princeton University, 41 Olden St, Princeton, NJ 08540, United States

*depablo@uchicago.edu 


\section{Contents:}

1. Synthesis Details

2. SEC Characterization

3. DSC Thermograms

4. Molecular Dynamics Simulation Protocols

5. Force Field Parameters for MD Simulations

6. Fictive Temperature Determination

7. Solvation-site Connectivity

8. Bond Vector Autocorrelation Functions

9. Fitting Parameters for Coordination Autocorrelation Functions

10. Radial Distribution Functions in Alternative Plot Range

11. Simulation Results at Lower Concentration

12. Simulation Results at Higher Concentration 


\section{Synthesis of POEM9}

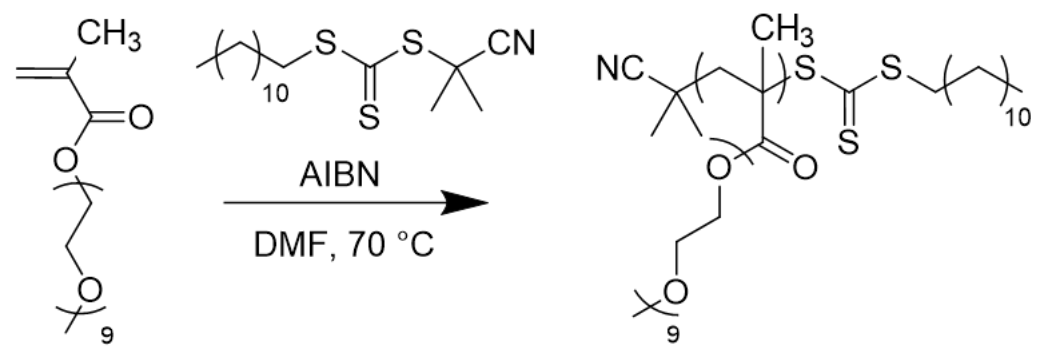

Scheme S1. Reaction scheme for RAFT polymerization of homopolymer POEM9. 


\section{SEC Characterization}

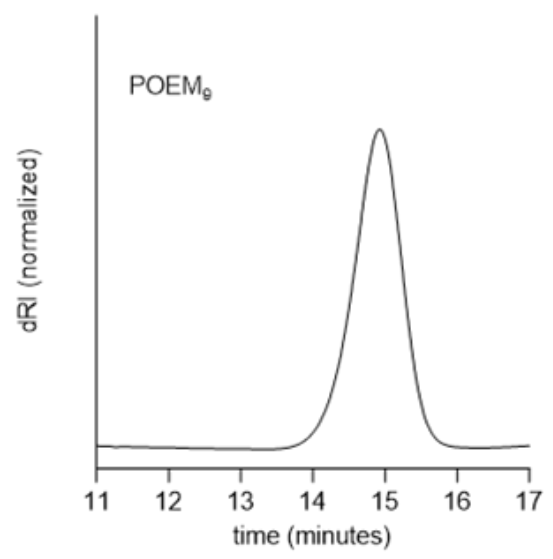

Fig. S1. Differential refractive index $(\mathrm{dRI}) v s$. elution time data taken from SEC-MALS measurements of synthesized polymers in THF. Molecular weight and dispersity are taken from this data assuming a differential refractive index of $d n / d c=0.073 \mathrm{~mL} \mathrm{~g}^{-1}$. 


\section{DSC Thermograms}

Calorimetric $T_{\mathrm{g}}$ of neat polymers and polymer electrolytes were determined by DSC using a TA Instruments Discovery 2500 DSC. $T_{\mathrm{g}}$ is reported as the inflection point of the heat flow thermograms taken from the third heating curve shown in Figure S2.

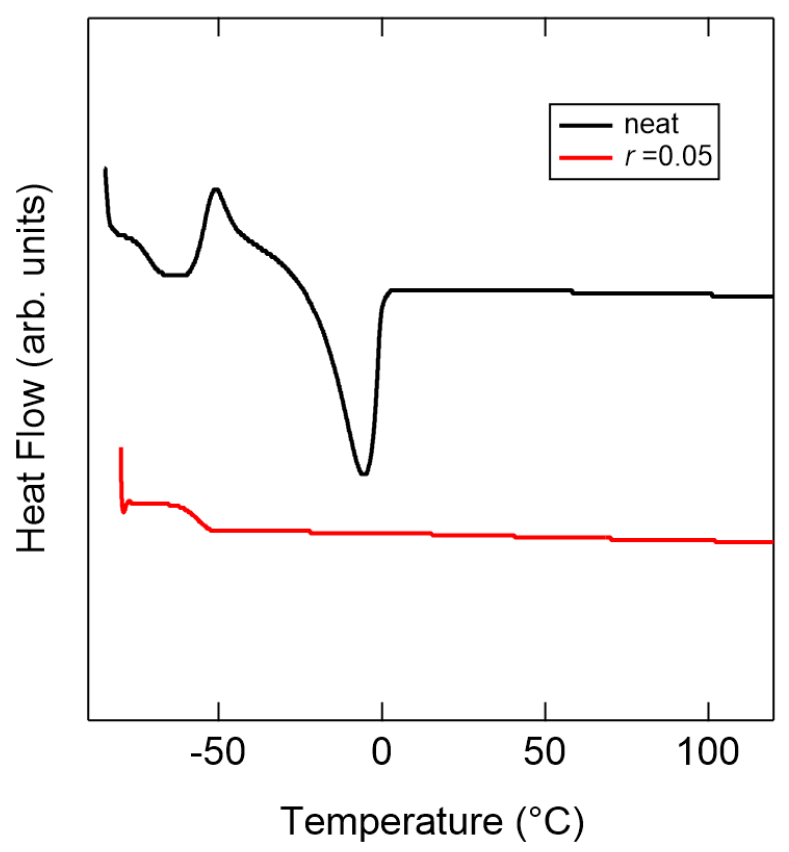

Fig. S2. DSC thermograms of neat and $r=0.05$ POEM samples. These curves represent the third heating cycle, and $T_{\mathrm{g}}$ is taken as the midpoint in the inflection in the heat flow rate. 


\section{Force Field Parameters for Molecular Dynamics Simulations}

In this study, the TraPPE-UA force field ${ }^{4-6}$ is used for all inter- and intramolecular interactions between polymer atoms. Instead of rigid bonds used in TraPPE-UA force field, the generalized CHARMM bonding parameters are used ${ }^{7}$. Parameters for the lithium cation are adapted from a previous simulation study ${ }^{8}$. Figure S3 provides reference labels for the different atom types in polymer for assigning the appropriate force field parameters.

\subsection{United Atom Model: Terminal Groups, Tacticity, and Atom Type Assignments}

The PEO chain has 3333 monomers $\left(146.8 \mathrm{k} \mathrm{g} \mathrm{mol}^{-1}\right)$. The POEM chain has 300 monomers $\left(155.4 \mathrm{k} \mathrm{g} \mathrm{mol}^{-1}\right)$. For PEO, the termini at both ends are methoxy end groups as noted in (OET-CE3) in Figure S3. For POEM, the termini at both ends are $\mathrm{CH} 3$ that are connected to a CT interaction site. POEM is this study is atactic. When generating the atactic POEM chain, the stereochemistry of each chiral center is randomly chosen as $\mathrm{S}$ or $\mathrm{R}$ with equal probability.

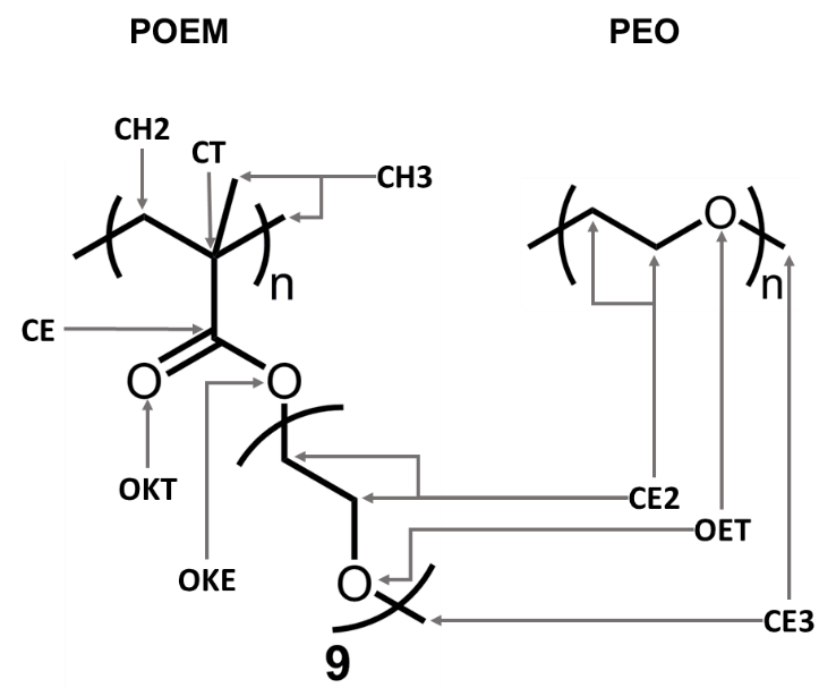

Figure S3. Reference labels for atom types in force field parameters. 


\subsection{Non-bonded Interaction Parameters}

The nonbonded interactions include Lennard-Jones interactions and Coulombic interactions. For united atoms $i$ and $j$, their nonbonded interaction is:

$$
u_{n b}\left(r_{i j}\right)=4 \varepsilon_{i j}\left[\left(\frac{\sigma_{i j}}{r_{i j}}\right)^{12}-\left(\frac{\sigma_{i j}}{r_{i j}}\right)^{6}\right]+\frac{q_{i} q_{j}}{4 \pi \epsilon_{0} r_{i j}}
$$

where $r_{i j}$ is the separation distance between atoms $i$ and $j, \sigma_{i j}$ is the Lennard-Jones diameter, $\varepsilon_{i j}$ is the LennardJones interaction strength, $\epsilon_{0}$ is the vacuum permittivity. $q_{i}$ and $q_{j}$ are the partial charges of atoms $i$ and $j$.

LJ interactions are computed with Lorentz-Berthelot mixing rules. For 1-2, 1-3, and 1-4 bonded interaction sites, the Lennard-Jones interactions are turned off. Coulomb interactions are turned off for 1-2 and 1-3 bonded interaction sites, and scaled by 0.5 for $1-4$ bonded interactions. The non-bonded interaction parameters are listed in table S1.

Table S1: Nonbonded interaction potential parameters for polymer atoms.

\begin{tabular}{l|cccc}
\hline \hline \multicolumn{1}{c|}{ atom } & $m(\mathrm{amu})$ & $\sigma_{i i}(\AA)$ & $\epsilon_{i i}\left(\frac{\mathrm{kcal}}{\mathrm{mol}}\right)$ & $q(e)$ \\
\hline $\mathrm{CE} 2$ & 14.027 & 3.950 & 0.091411 & 0.25 \\
$\mathrm{CE} 3$ & 15.035 & 3.750 & 0.194746 & 0.25 \\
OET & 15.999 & 2.850 & 0.109296 & -0.50 \\
$\mathrm{CH} 2$ & 14.027 & 3.950 & 0.091411 & 0.00 \\
$\mathrm{CH} 3$ & 15.035 & 3.750 & 0.194746 & 0.00 \\
$\mathrm{CT}$ & 12.011 & 6.400 & 0.000994 & 0.05 \\
$\mathrm{CE}$ & 12.011 & 3.820 & 0.079488 & 0.55 \\
$\mathrm{OKT}$ & 15.999 & 3.050 & 0.156989 & -0.45 \\
OKE & 15.999 & 2.800 & 0.109296 & -0.40 \\
\hline
\end{tabular}




\subsection{Bonding Potential Parameters}

1-2 bonded united atoms interact via a harmonic bonding potential in the form:

$$
u_{\text {bond }}\left(r_{i j}\right)=k_{\text {bond }}\left(r_{i j}-r_{i j}^{(0)}\right)^{2}
$$

Where $r_{i j}$ is the separation distance between atoms $i$ and $j, k_{b o n d}$ is the force constant and $r_{i j}{ }^{(0)}$ is the equilibrium bond length. The bonding interaction parameters are listed in table $\mathrm{S} 2$.

Table S2: Bonding potential parameters for polymer atoms.

\begin{tabular}{c|cc|l|cc}
\hline \hline bond & $k_{\text {bond }}\left(\frac{\mathrm{kcal}}{\mathrm{mol} \cdot \AA^{2}}\right)$ & $r_{i j}{ }^{(0)}(\AA)$ & bond & $k_{\text {bond }}\left(\frac{\mathrm{kcal}}{\mathrm{mol} \cdot \AA^{2}}\right)$ & $r_{i j}{ }^{(0)}(\AA)$ \\
\hline CE2-CE2 & 225.0 & 1.54 & CE2-OKE & 360.0 & 1.41 \\
CE2-OET & 360.0 & 1.41 & CE-CT & 200.0 & 1.52 \\
CE3-OET & 360.0 & 1.41 & CE-OKT & 360.0 & 1.20 \\
CH3-CT & 225.0 & 1.54 & CE-OKE & 360.0 & 1.34 \\
CH2-CT & 225.0 & 1.54 & CH2-CH2 & 225.0 & 1.54 \\
\hline
\end{tabular}




\subsection{Bending Potential Parameters}

1-2-3 bonded united atoms interact via a harmonic bending potential in the form:

$$
u_{\text {bend }}\left(\theta_{i j k}\right)=k_{\text {bend }}\left(\theta_{i j k}-\theta_{i j k}^{(0)}\right)^{2} \text {, }
$$

Where $k_{\text {bend }}$ is the force constant, $\theta_{i j k}$ is the angle between united atoms $i, j$, and $k$, and $\theta_{i j k}{ }^{(0)}$ is the equilibrium angle. The bending interaction parameters are listed in table S3.

Table S3: Bending potential parameters for polymer atoms.

\begin{tabular}{c|cc||c|cc}
\hline \hline bend & $k_{\text {bend }}\left(\frac{\mathrm{kcal}}{{\mathrm{mol} \cdot \mathrm{rad}^{2}}^{2}}\right)$ & $\theta_{i j k}{ }^{(0)}(\AA)$ & bend & $k_{\text {bend }}\left(\frac{\mathrm{kcal}}{\mathrm{mol}^{\mathrm{rad}}{ }^{2}}\right)$ & $\theta_{i j k}{ }^{(0)}(\AA)$ \\
\hline CE2-OET-CE2 & 60.0136 & 112.0 & OKT-CE2-CE2 & 49.9782 & 112.0 \\
CE2-OET-CE3 & 60.0136 & 112.0 & CH2-CT-CE & 62.1000 & 109.5 \\
CE2-CE2-OET & 49.9782 & 112.0 & CT-CE-OKT & 62.1000 & 125.0 \\
CH2-CT-CH2 & 62.1000 & 109.5 & CT-CE-OKE & 70.1483 & 110.0 \\
CH3-CT-CH2 & 62.1000 & 109.5 & CH3-CT-CE & 62.1000 & 109.5 \\
CT-CH2-CT & 62.1000 & 114.0 & CH3-CT-CH3 & 62.1000 & 109.5 \\
CE-OKE-CE2 & 62.1000 & 115.0 & OKE-CE2-CE2 & 49.9782 & 112.0 \\
OKT-CE-OKE & 62.1000 & 125.0 & & & \\
\hline
\end{tabular}




\subsection{Torsional Potential Parameters}

The torsional potentials for 1-2-3-4 bonded united atoms take the form:

$$
\begin{aligned}
u_{\text {tors }}\left(\phi_{i j k l}\right)= & \frac{K_{1}}{2}\left[1+\cos \left(\phi_{i j k l}\right)\right]+\frac{K_{2}}{2}\left[1-\cos \left(2 \phi_{i j k l}\right)\right]+ \\
& \frac{K_{3}}{2}\left[1+\cos \left(3 \phi_{i j k l}\right)\right]+\frac{K_{4}}{2}\left[1-\cos \left(4 \phi_{i j k l}\right)\right],
\end{aligned}
$$

Where $c_{1}, c_{2}$, and $c_{3}$ are coefficients and $\phi_{i j k l}$ is the dihedral angle defined by atoms $i, j, k$, and $l$. The torsional interaction parameters are listed in table S4.

Table S4: Torsional potential parameters for polymer atoms.

\begin{tabular}{c|cccc}
\hline \hline torsion & $K_{1}\left(\frac{\mathrm{kcal}}{\mathrm{mol}}\right)$ & $K_{2}\left(\frac{\mathrm{kcal}}{\mathrm{mol}}\right)$ & $K_{3}\left(\frac{\mathrm{kcal}}{\mathrm{mol}}\right)$ & $K_{4}\left(\frac{\mathrm{kcal}}{\mathrm{mol}}\right)$ \\
\hline OKE-CE2-CE2-OET & 1.888000 & -1.888000 & 0.000000 & 0.000000 \\
OET-CE2-CE2-OET & 1.888000 & -1.888000 & 0.000000 & 0.000000 \\
CE2-CE2-OET-CE2 & 2.882840 & -0.650809 & 2.218510 & 0.000000 \\
CE2-CE2-OET-CE3 & 2.882840 & -0.650809 & 2.218510 & 0.000000 \\
CH3-CT-CH2-CT & 0.000000 & 0.000000 & 1.833350 & 0.000000 \\
CH2-CT-CH2-CT & 0.000000 & 0.000000 & 1.833350 & 0.000000 \\
CT-CH2-CT-CE & 0.000000 & 0.000000 & 1.833350 & 0.000000 \\
CH3-CT-CE-OKT & -0.919280 & 0.229880 & -0.609280 & 0.000000 \\
CH2-CT-CE-OKT & -0.919280 & 0.229880 & -0.609280 & 0.000000 \\
CH3-CT-CE-OKE & 0.915310 & 0.216840 & 0.609670 & 0.000000 \\
CH2-CT-CE-OKE & 0.915310 & 0.216840 & 0.609670 & 0.000000 \\
CT-CE-OKE-CE2 & 9.689610 & 7.678560 & 1.387070 & 0.000000 \\
CE-OKE-CE2-CE2 & -2.988080 & 2.142010 & -0.087834 & 0.203768 \\
\hline
\end{tabular}




\section{Simulation Protocol}

To prepare the starting configuration, a single polymer chain with approximately 10,000 interaction sites is initiated in a large box. The polymer chain is first relaxed under NVT ensemble at $500 \mathrm{~K}$ for $30 \mathrm{~ns}$. Then a 20 ns NPT equilibration at $500 \mathrm{~K}$ and 1 bar is performed to ensure the density does not drift. The process of introduction of $\mathrm{Li}^{+}$is as follows: $\mathrm{Li}^{+}$and $\mathrm{TFSI}^{-}$ions are introduced at random positions in the equilibrated neat polymer at the designated ratio of $r=0.05=[\mathrm{LiTFSI}] /[\mathrm{EO}]$. To remove any steric clashes in the simulation cell, the system is relaxed under NVE ensemble for 10,000 steps, where the maximum displacement of an atom is limited to $0.1 \AA$ A per step. The system is subsequently equilibrated for $15 \mathrm{~ns}$ under NPT at $400 \mathrm{~K}$ and 1 bar. To perform simulation at each temperature, the salt-doped polymer is equilibrated at the designated temperature under NPT for $10 \mathrm{~ns}$. Then the system is further equilibrated for $10 \mathrm{~ns}$ under NVT ensemble. In the beginning of this NVT equilibration, the box size is scaled to reflect the ensemble average density during at the last $3 \mathrm{~ns}$ of NPT equilibration. Then, the trajectory for analysis is collected from a 100-ns production simulation under NVT ensemble. 


\section{Fictive Temperature Determination}

To determine the fictive temperature, the polymer is cooled from $400 \mathrm{~K}$ to $100 \mathrm{~K}$ at a cooling rate of $10 \mathrm{~K} / \mathrm{ns}$ at 1 bar. The density is plotted as a function of temperature under NPT ensemble. Two linear segments are fitted to for the glass and liquid region respectively. The $T_{g}$ is determined as the intercept of the extrapolated linear fit lines. This procedure to calculate $T_{g}$ is sensitive to the fitting range of the two segments. Therefore, the end points of the fitting segments are systematically sampled in the following manner. The starting point of the glassy segment was fixed at $50 \mathrm{~K}$, while the end point of the glassy segment was randomly sampled between $180 \mathrm{~K}$ and $200 \mathrm{~K}$. Similarly, the starting point of the liquid segment was fixed at $400 \mathrm{~K}$, whereas the end point of the liquid segment was sampled between $300 \mathrm{~K}$ and $320 \mathrm{~K}$. The fitting intervals for the glassy segment and the liquid segment are randomly drawn within the sampling interval defined above, the intersections between which yield a set of $T_{g}$ values. Only $T_{g}$ values that fall between the end points of fitting segments are kept. The $T_{g}$ calculation described above was repeated for another independent sample. For each sample, combinations of glassy and liquid fitting ranges are generated 10,000 times. The mean and standard deviation of the set of $T_{g}$ values collected from both samples are reported. Figure S4 includes the cooling curve and the distribution of resulting $T_{g}$ values collected. 
(a)

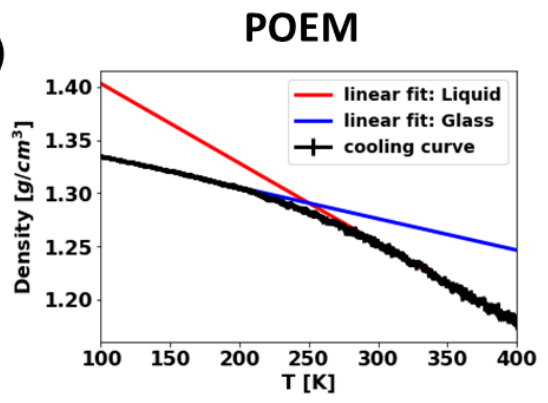

(b)

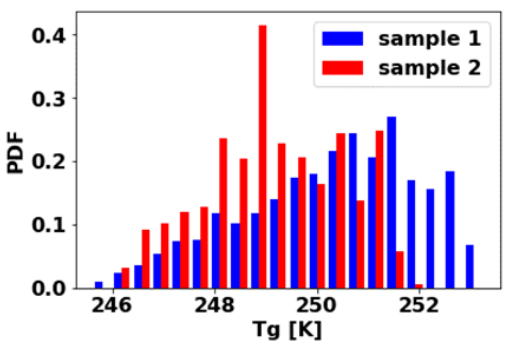

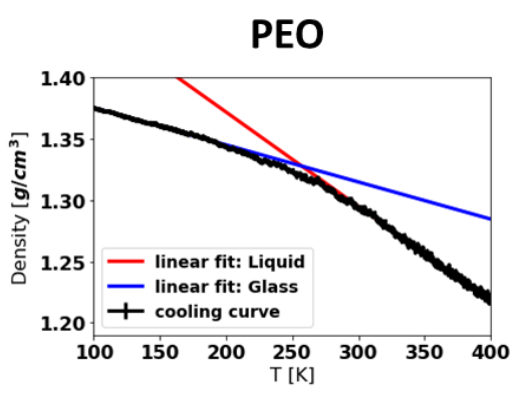

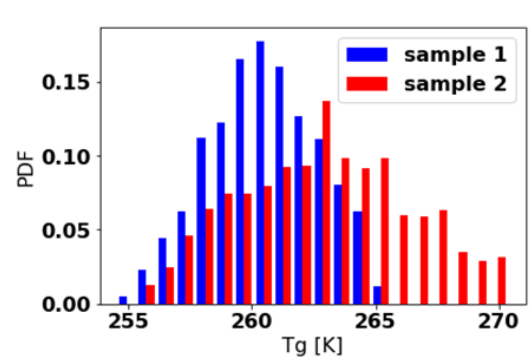

Figure S4. Fictive temperatures in simulations. (a) Representative density as a function of temperature. Example fitting lines for liquid and glassy regions are shown in red and blue, respectively. The fitting range for the example linear fits are $[100 \mathrm{~K}, 200 \mathrm{~K}]$ for the glassy region and $[300 \mathrm{~K}, 400 \mathrm{~K}]$ for the liquid region. (b) Normalized histograms of collected $T_{g}$ values. Histograms from different samples are plotted in different colors. 


\section{Solvation-site Connectivity}

The solvation-site connectivity is calculated as:

$$
\kappa=e^{-\left\langle r_{n n}\right\rangle}
$$

Where $\left\langle r_{n n}\right\rangle$ is the average nearest neighbor distances among possible solvation sites ${ }^{9,10}$. The values for solvation-site connectivity for POEM and PEO and their respective ratio are listed in Table S5 at each reduced temperature. The mean and uncertainty in solvation-site connectivity are obtained by averaging over 100 frames sampled from each trajectory.

Table S5. Solvation-site connectivity values for PEO and POEM at $T-T_{g}=50,150$, and $250 \mathrm{~K}$.

\begin{tabular}{c|ccc}
\hline & $T-T_{g}=50 \mathrm{~K}$ & $T-T_{g}=150 \mathrm{~K}$ & $T-T_{g}=250 \mathrm{~K}$ \\
\hline$\kappa_{P E O}$ & $(1.8 \pm 0.19) \times 10^{-3}$ & $(1.3 \pm 0.14) \times 10^{-3}$ & $(8.9 \pm 1.2) \times 10^{-4}$ \\
$\kappa_{P O E M}$ & $(1.1 \pm 0.15) \times 10^{-3}$ & $(7.0 \pm 1.2) \times 10^{-4}$ & $(5.2 \pm 0.91) \times 10^{-4}$ \\
$\frac{\kappa_{P O E M}}{\kappa_{P E O}}$ & $0.58 \pm 0.10$ & $0.55 \pm 0.11$ & $0.58 \pm 0.13$ \\
\hline
\end{tabular}




\section{Bond Vector Autocorrelation Functions}

\subsection{BVAF Averaged Over All Ether Oxygens:}

Mean relaxation times plotted in Figure 3D (solid blue and red lines) are obtained by computing the first moment of the stretched exponential decay fitted to the BVAFs. In each system, this BVAF is calculated for all C-O(ether) bond vectors regardless of the EO positions. Figure S5 shows the original BVAFs and the corresponding stretched exponential fits.
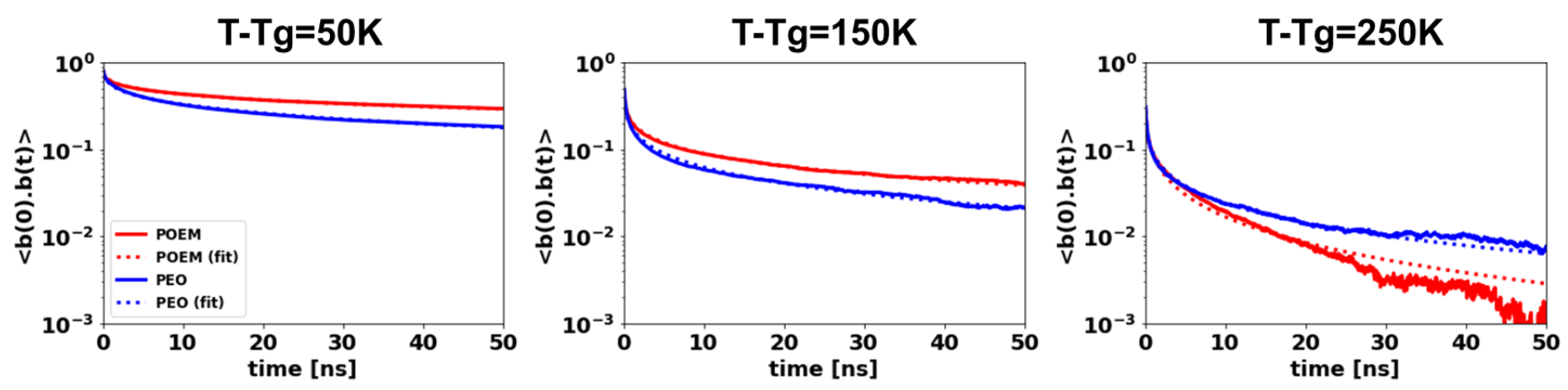

Figure S5: Bond vector autocorrelation functions (BVAF) for ether oxygens in PEO and POEM and corresponding stretched exponential fitting lines at fixed reduced temperatures $(50 \mathrm{~K}, 150 \mathrm{~K}$, and $250 \mathrm{~K}$ above $\left.T_{g}\right)$

\subsection{BVAFs of Ether Oxygens Along Sidechain}

Mean relaxation times plotted in Figure 3B (scattered points) are obtained by computing the first moment of the stretched exponential decay fitted to the BVAFs of ether oxygens at each position along the sidechain. Figure S6 shows the original BVAFs. For clarity, the stretched exponential fits are omitted. 

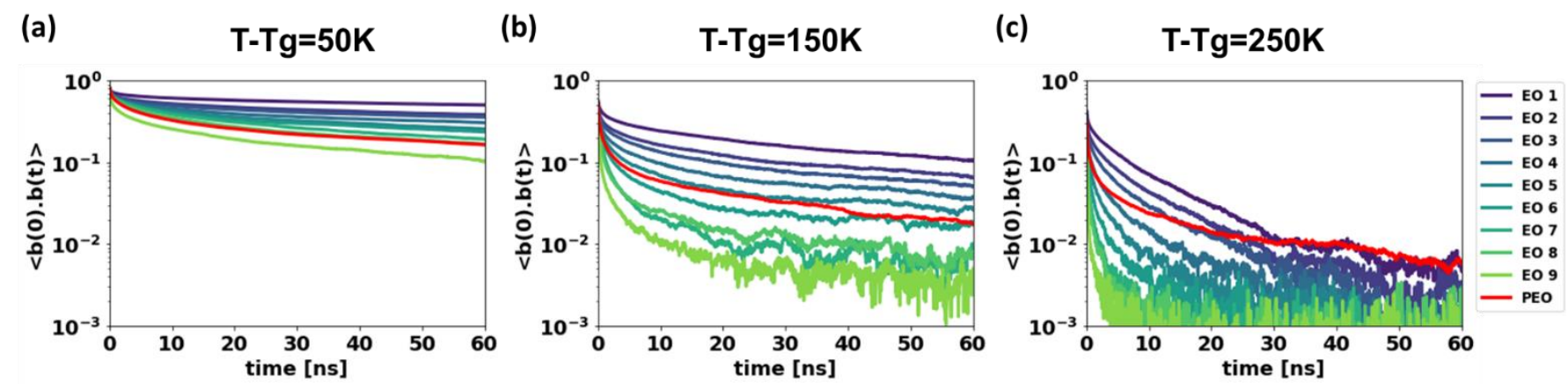

Figure S6: Bond rotation autocorrelation of different ether oxygen along the POEM side chains. EO 1

corresponds to the ether oxygens that are closest to the backbone. EO 9 corresponds to oxygens that are at the tail of the sidechains. For reference, the red line is the bond rotation autocorrelation function averaged over all ether oxygens in PEO system. The temperatures are $50 \mathrm{~K}, 150 \mathrm{~K}$, and $250 \mathrm{~K}$ above $T_{g}$ respectively. 


\section{Fitting Parameters for Coordination Autocorrelation Functions}

Table S6 Stretched exponential fitting parameters for CACF.

\begin{tabular}{c|ccc}
\hline \hline CACF & $\beta$ & $\tau(\mathrm{ns})$ & $\langle\tau\rangle(\mathrm{ns})$ \\
\hline PEO combined & 0.33 & 1.38 & 9.0 \\
PEO interchain & 0.41 & 3.76 & 11.6 \\
POEM combined & 0.51 & 22.14 & 42.4 \\
POEM interchain & 0.82 & 38.22 & 42.7 \\
\hline
\end{tabular}




\section{Radial Distribution Functions in Alternative Plot Range}
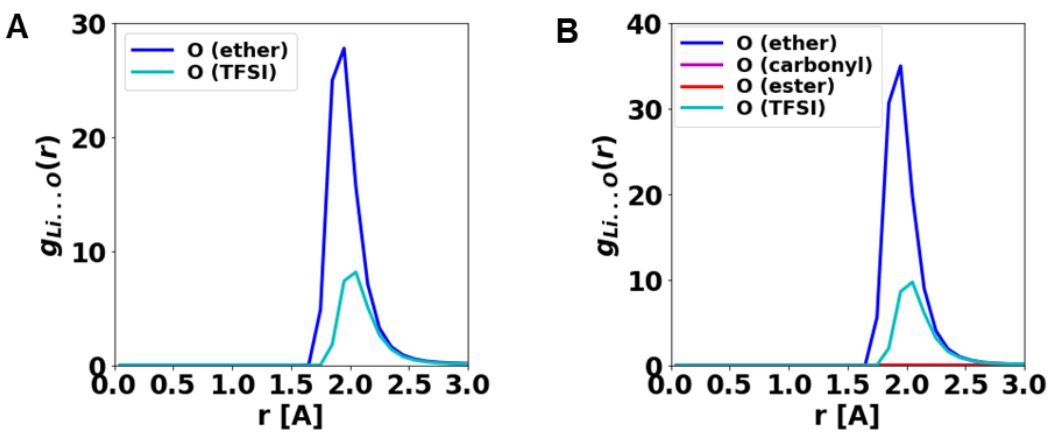

Figure S7: Radial distribution functions between $\mathrm{Li}^{+}$and oxygen atoms in (A) PEO and (B) POEM at $150 \mathrm{~K}$ above $T_{g}$ shown. Figure 3A-B are plotted here with a smaller x-axis range to highlight the positions of the first RDF peaks and an extended y-axis range to highlight the magnitudes of the first PDF peaks. 


\section{Simulation Results at Lower Concentration}

The PEO and POEM systems are further examined computationally at salt concentration $r=0.025$ (Li:EO = 1:40). Using the same $T_{g}$ calculation protocol, the $T_{g}$ 's of PEO and POEM at $r=0.025$ are estimated to be $241 \pm 1$ $\mathrm{K}$ and $241 \pm 2 \mathrm{~K}$, respectively.

The solvation behavior analysis presented in Figure $\mathrm{S} 8$ is conducted at $150 \mathrm{~K}$ above $T_{g}$. The $\mathrm{Li}^{+}-\mathrm{O}$ RDF indicate that the locations of each Li-O peaks at $r=0.025$ are the same as those at $r=0.05$ (Figure 3), while the magnitudes of the peaks shift upwards because at $r=0.025$ the RDF are normalized by a lower concentration. Figure S8D shows that the top five populated coordination motifs at $r=0.025$ are in the same order as those at $r=0.05$ (Figure 4A), where $\mathrm{Li}^{+}$are predominately coordinated by six EOs. In PEO, the six EOs belong to a single contiguous chain, whereas in POEM they come from two separate chains. Figure S8D presents the $\mathrm{Li}^{+}$binding probability of EOs at each position along the POEM sidechains at $r=0.025$. It indicates that the observed $\mathrm{Li}^{+}$solvating behavior is consistent between the two concentrations $r=0.025$ and 0.05 , where $\mathrm{Li}^{+}$solvation is relatively localized at EOs at positions 4-7. Overall, Figure S8 shows that the solvation behavior at $r=0.05$ presented in the paper is still valid at $r=0.025$.
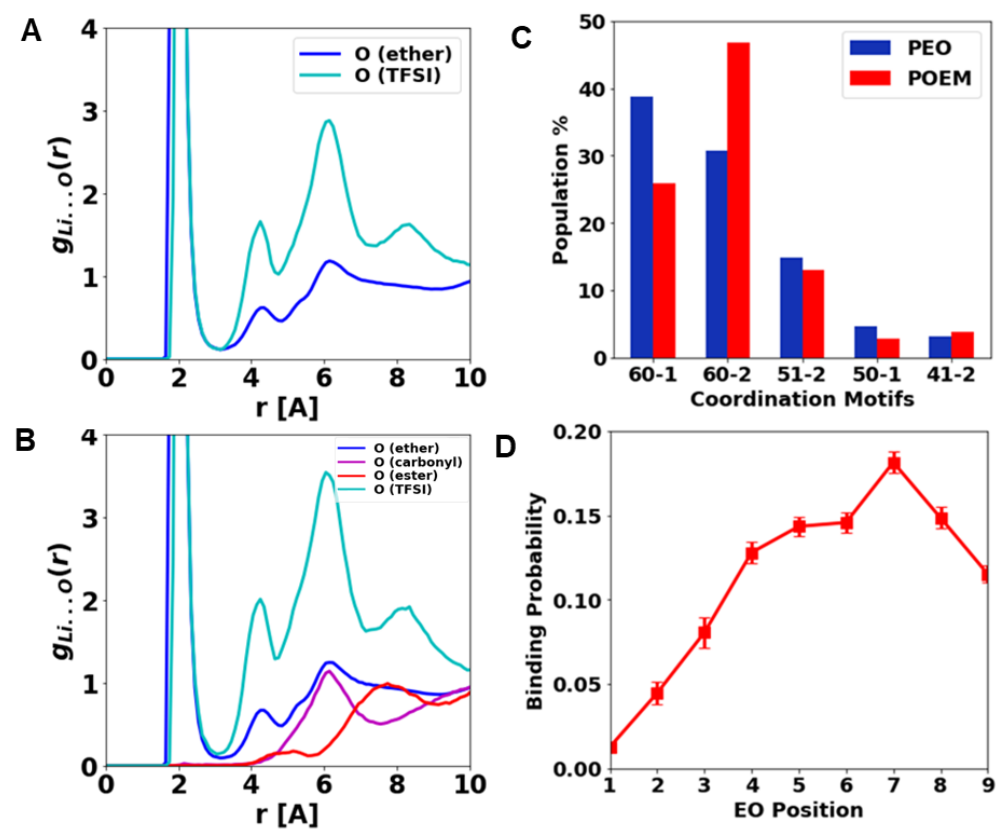

Fig. S8. Solvation behavior in PEO and POEM at $T-T_{\mathrm{g}}=150 \mathrm{~K}$ and $r=0.025$. Radial distribution functions between $\mathrm{Li}^{+}$and oxygen atoms in (A) PEO and (B) POEM at $150 \mathrm{~K}$ above $\mathrm{T}_{\mathrm{g}}$. The RDFs are normalized by the concentration of each oxygen type. (C) Population of $\mathrm{Li}^{+}$coordination motifs in PEO and POEM. Only the five 
most abundant coordination motifs are shown. (D) Binding probability of EOs to $\mathrm{Li}^{+}$as a function of position along a POEM sidechain.
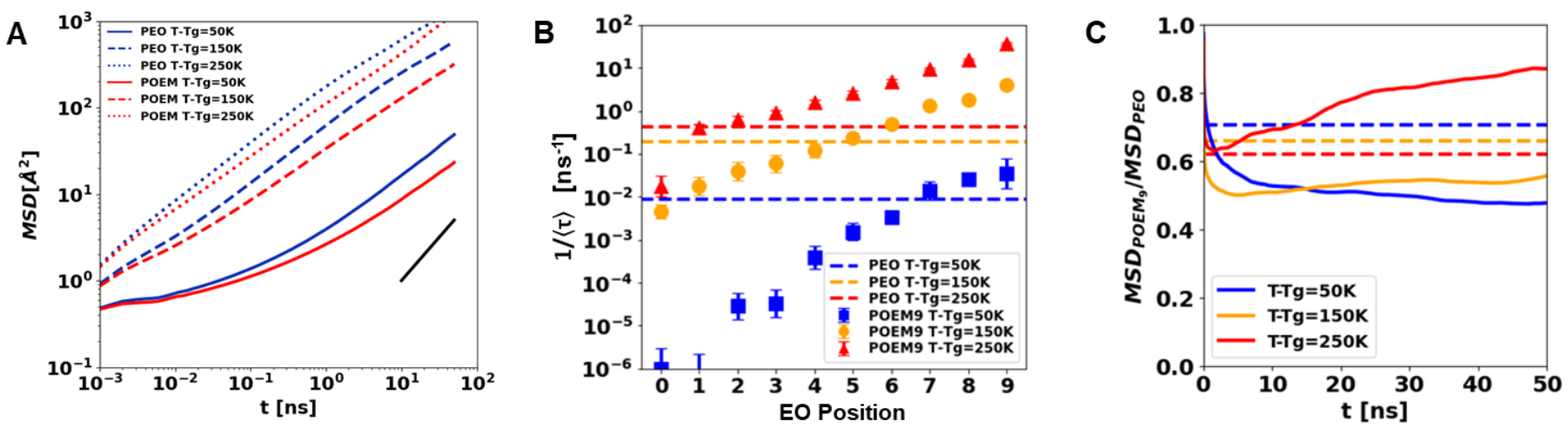

Figure S9. Characterization of temperature-dependent ion and chain mobility in PEO and POEM at $r=$

0.025. (A) Simulated MSD of $\mathrm{Li}^{+}$in PEO and POEM at fixed reduced temperatures for $r=0.025$ electrolytes. The MSD was computed up to 50 ns using 120-ns trajectories. The black segment represents a guideline with unit slope. (B) $1 /<\tau>$ extracted from BVAFs as a function of EO position along the POEM sidechain at three fixed $T-T_{\mathrm{g}}$. The dashed lines are inverse relaxation times $(1 /<\tau>)$ for all EOs in PEO system at various relative temperatures. (C) Normalized MSD of $\mathrm{Li}^{+}$in POEM at fixed $T-T_{\mathrm{g}}$. The dashed lines are ratios of solvation site connectivity at different temperatures.

Figure S9 compares the ion mobility and segmental dynamics between PEO and POEM at three reduced temperatures. The $\mathrm{Li}^{+}$mobility indicated by MSD and the chain mobilities indicated by BVAF are faster at the lower concentration of $r=0.025$ compared to $r=0.05$ presented in the paper. The position dependency of sidechain EO mobilities reported at $r=0.05$ is also observed at $r=0.025$. The comparison among relative $\mathrm{Li}^{+}$ mobility, relative chain mobilities and the relative connectivity are consistent with the case at $r=0.05$, except for the crossover temperature, the temperature at which $\mathrm{Li}^{+}$in POEM benefit from the improved chain mobility such that the $\mathrm{Li}^{+}$mobility starts to outperform what would be expected based on the relative connectivity. As reported in the paper, such temperature for $r=0.05$ is approximately $150 \mathrm{~K}$ above $T_{g}$. Here at $r=0.025$, the crossover temperature seems to fall between $150 \mathrm{~K}$ and $250 \mathrm{~K}$ above $T_{\mathrm{g}}$. Overall, Figure $\mathrm{S} 9$ shows that the 
conclusions regarding $\mathrm{Li}^{+}$and chain mobilities at $r=0.05$ presented in the paper is consistent with results at $r=0.025$. 


\section{Simulation Results at Higher Concentration}

The PEO and POEM systems are additionally examined computationally at salt concentration $r=0.1$ (Li:EO = 1:10). This concentration is slightly higher than the widely used concentration, $r=0.085$ (Li:EO 1:12), in experimental studies. The simulations are conducted at temperatures relative to $T_{g}$ 's obtained at $r=0.05$, which are noted as $T_{g}^{*}$ 's in the following text. We believe this would still yield qualitatively reliable results.

The solvation behavior analysis presented in Figure $\mathrm{S} 10$ is conducted at $150 \mathrm{~K}$ above $T_{g}{ }^{*}$. The $\mathrm{Li}^{+}-\mathrm{O} \mathrm{RDF}$ in Figure S10 indicate that the locations of first and second Li-EO peaks at $r=0.1$ are the same as those at $r=0.05$ (Figure 3). However, the third $\mathrm{Li}^{+}$-EO peak seen at $r=0.05$ at approximately $6 \AA$ is not present at $r=0.1$. Moreover, at $r=0.1$, the Li-O(carbonyl) and Li-O(ester) RDF exhibit small peaks at around $2 \AA$. This suggests that carbonyl oxygens and ester oxygens start to participate in $\mathrm{Li}^{+}$coordination at $r=0.1$, a phenomenon unseen at $r=0.05$. These $\mathrm{RDF}$ suggest that at a salt concentration as high as $r=0.1, \mathrm{Li}^{+}$in POEM can adopt additional solvation structures that do not exist in PEO. The top five populated coordination motifs at $r=0.1$ are presented in Figure S10C. It shows that $\mathrm{Li}^{+}$solvation is no longer dominated by six-EO motifs. Rather, a diverse range of coordination motifs are observed, without any obviously dominant one. Additionally, there seems to be more participation from oxygens from $\mathrm{TFSI}^{-}$, a reasonable observation considering the high salt concentration. Figure S10D presents the $\mathrm{Li}^{+}$binding probability of EOs at each position along the POEM sidechains at $r=0.1$. The distribution of binding probability is qualitatively different from results at the two lower concentrations tested, where $\mathrm{Li}^{+}$solvation is relatively localized at EOs at positions 4-7. At $r=0.1$, there is increased coordination of $\mathrm{Li}^{+}$by EOs that are closest to the backbone, consistent with the suggestion from RDF that carbonyl oxygens and ester oxygens start to participate in $\mathrm{Li}^{+}$solvation at this salt concentration. Overall, Figure $\mathrm{S} 10$ shows that at increased salt concentration as high as $r=0.1$, the $\mathrm{Li}^{+}$solvation environment is different. Most importantly, the difference in more prominent in POEM than in PEO, since there are additional $\mathrm{Li}^{+}$solvation structures involving ester and carbonyl oxygens apartfrom those involving only EOs observed at lower concentrations. The difference in solvation environment could lead to different transport mechanism at elevated salt concentrations. $\mathrm{Li}^{+}$transport in POEM at concentrated conditions may have very rich and diverse behavior, and thereby worth investigating. 

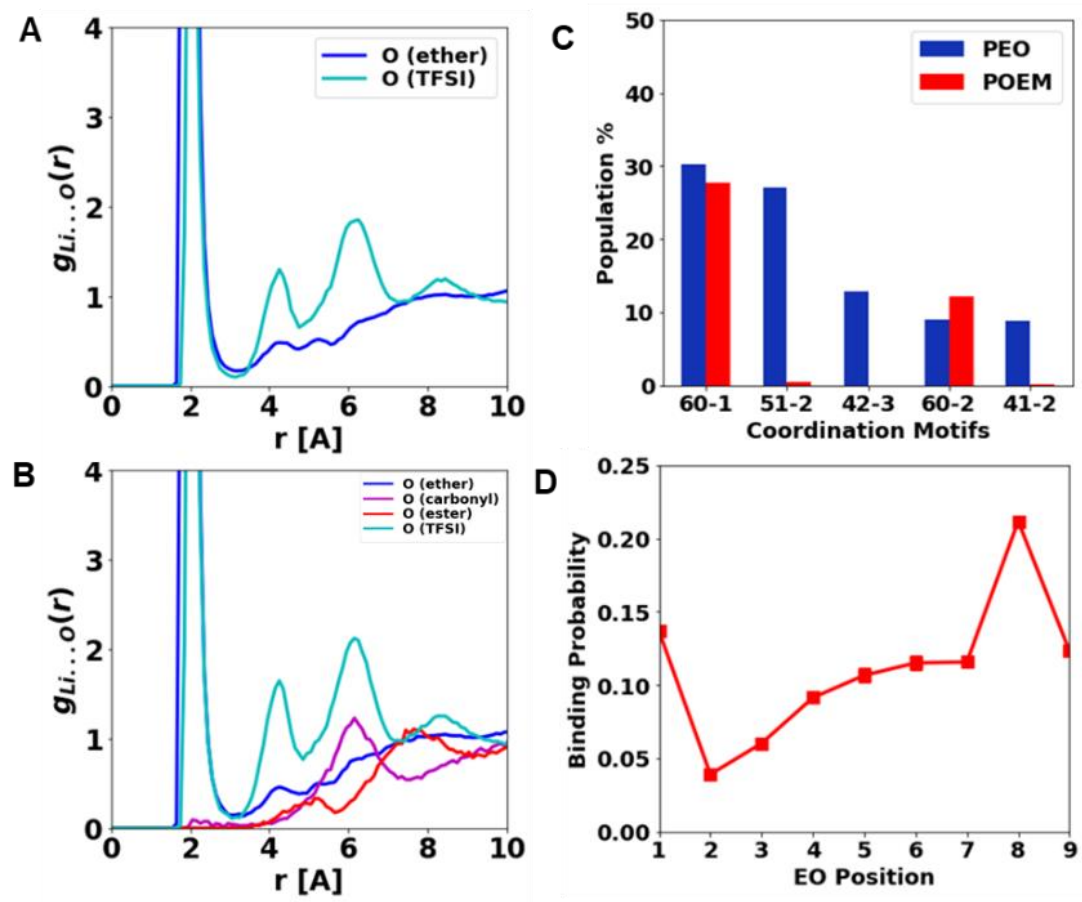

Fig. S10. Solvation behavior in PEO and POEM at $T-T_{\mathrm{g}}{ }^{*}=150 \mathrm{~K}$ and $r=0.1$. Radial distribution functions between $\mathrm{Li}^{+}$and oxygen atoms in (A) PEO and (B) POEM at $150 \mathrm{~K}$ above $T_{g}{ }^{*}$. The RDFs are normalized by the concentration of each oxygen type. (C) Population of $\mathrm{Li}^{+}$coordination motifs in PEO and POEM. Only the five most abundant coordination motifs are shown. (D) Binding probability of EOs to $\mathrm{Li}^{+}$as a function of position along a POEM sidechain.
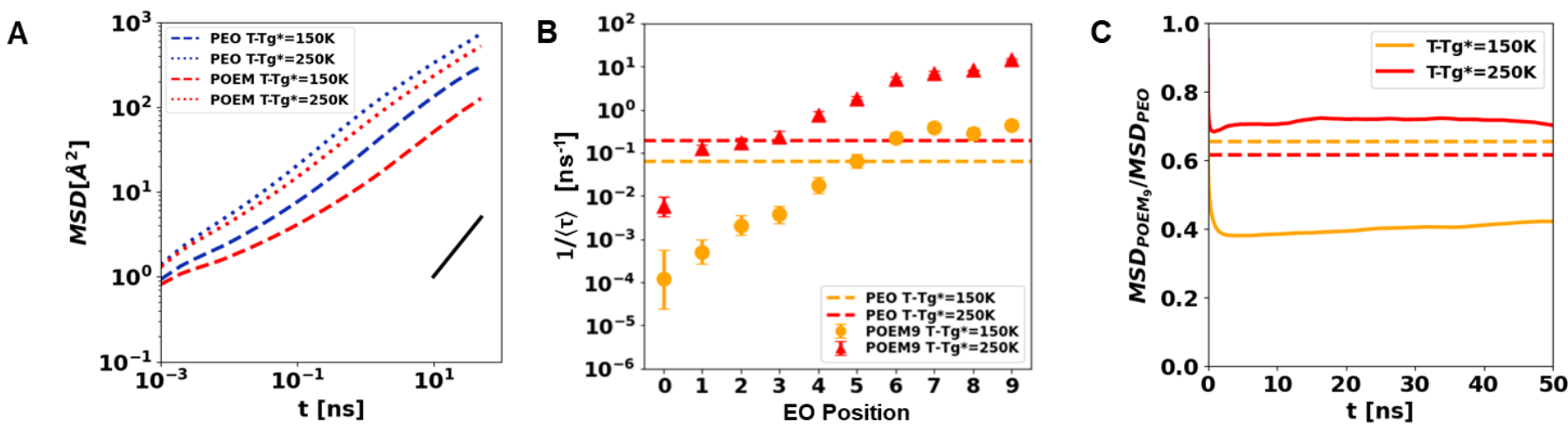

Figure S11. Characterization of temperature-dependent ion and chain mobility in PEO and POEM at $r$ = 0.1. (A) Simulated MSD of $\mathrm{Li}^{+}$in PEO and POEM at fixed reduced temperatures for $r=0.1$ electrolytes. The MSD was computed up to $50 \mathrm{~ns}$ using 120-ns trajectories. The black segment represents a guideline with unit slope. (B) $1 /<\tau>$ extracted from BVAFs as a function of EO position along the POEM sidechain at $T-T_{\mathrm{g}}{ }^{*}=150 \mathrm{~K}$ and $250 \mathrm{~K}$. The dashed lines are inverse relaxation times $(1 /<\tau>)$ for all EOs in PEO system at various relative 
temperatures. An EO position of 1 refers to the EO that is closest to the backbone. The EO position of " 0 " refers to the $1 /<\tau>$ calculated for the C-C bonds along the backbone. $(\mathbf{C})$ Normalized MSD of $\mathrm{Li}^{+}$in POEM at $T-T_{\mathrm{g}}{ }^{*}=150$ $\mathrm{K}$ and $250 \mathrm{~K}$. The dashed lines are ratios of solvation site connectivity at different temperatures.

Figure S11 compares the ion mobility and segmental dynamics between PEO and POEM at two reduced temperatures. Due to the slow dynamics, the simulations are conducted only at $150 \mathrm{~K}$ and $250 \mathrm{~K}$ above $T_{g}{ }^{*}$. The $\mathrm{Li}^{+}$mobility indicated by MSD and the chain mobilities indicated by BVAF are slower at $r=0.1$ compared to $r=0.05$ presented in the paper. Similar to results at $r=0.05$, sidechain EOs also exhibit a separation in relaxation time scales at $r=0.1$. Based on the position dependent sidechain ion binding probabilities (Figure S10D) and mobilities (Figure S11B), the effective mobility of POEM is expected to be lower than PEO at $150 \mathrm{~K}$ above $T_{g}{ }^{*}$ and higher than PEO at $250 \mathrm{~K}$ above $T_{g}{ }^{*}$. Such result suggests that the effective chain mobilities, not $T_{g}$, can qualitatively capture the difference between relative $\mathrm{Li}^{+}$mobility and relative solvation site connectivity. Overall, Figure $\mathrm{S} 11$ shows that the comparison among relative $\mathrm{Li}^{+}$mobility, relative chain mobilities and the relative connectivity at $r=0.1$ is qualitatively similar as that at $r=0.05$. Based on the characterization of $\mathrm{Li}^{+}$ solvation behavior at $r=0.1$, we expect that additional factors due to the different solvation behavior and its corresponding transport mechanism need to be considered to fully capture the complete relationship among ion mobility, polymer properties, and polymer-ion interaction. The results further suggest that at salt concentration as high as $r=0.1$, there might also exist ion correlation effects, which requires a more detailed representation, such as polarization force field, to model.

Comparing among the three concentrations tested, we find that as salt concentration increases, an increase in number of carriers can be offset by a decrease in $\mathrm{Li}^{+}$mobilities. This suggests that there exists an optimal salt concentration for the conductivity performance of the material, at which there is a balance between increased number of carriers and compromised $\mathrm{Li}^{+}$mobilities. Moreover, further raising the concentration to $r=0.1$ can qualitatively alter the $\mathrm{Li}^{+}$solvation environment, which has been shown to be an important factor that determines the $\mathrm{Li}^{+}$transport mechanisms. Overall, results at various salt concentrations presented here provide additional insights into further investigations on $\mathrm{Li}^{+}$transport at concentrated conditions. 


\section{References}

(1) Hussain, H.; Mya, K. Y.; He, C. Self-Assembly of Brush-like Poly[Poly(Ethylene Glycol) Methyl Ether Methacrylate] Synthesized via Aqueous Atom Transfer Radical Polymerization. Langmuir 2008, 24 (23), 13279-13286. https://doi.org/10.1021/la802734e.

(2) Sharon, D.; Bennington, P.; Dolejsi, M.; Webb, M. A.; Dong, B. X.; de Pablo, J. J.; Nealey, P. F.; Patel, S. N. Intrinsic Ion Transport Properties of Block Copolymer Electrolytes. ACS Nano 2020, acsnano.0c03713. https://doi.org/10.1021/acsnano.0c03713.

(3) Dong, B. X.; Bennington, P.; Kambe, Y.; Sharon, D.; Dolejsi, M.; Strzalka, J.; Burnett, V. F.; Nealey, P. F.; Patel, S. N. Nanothin Film Conductivity Measurements Reveal Interfacial Influence on Ion Transport in Polymer Electrolytes. Mol. Syst. Des. Eng. 2019, 4 (3), 597-608. https://doi.org/10.1039/c9me00011a.

(4) Martin, M. G.; Siepmann, J. I. Transferable Potentials for Phase Equilibria. 1. United-Atom Description of n-Alkanes. J. Phys. Chem. B 1998, 102 (14), 2569-2577. https://doi.org/10.1021/jp972543+.

(5) Stubbs, J. M.; Potoff, J. J.; Siepmann, J. I. Transferable Potentials for Phase Equilibria. 6. United-Atom Description for Ethers, Glycols, Ketones, and Aldehydes. J. Phys. Chem. B 2004, 108 (45), 1759617605. https://doi.org/10.1021/jp049459w.

(6) Maerzke, K. A.; Schultz, N. E.; Ross, R. B.; Siepmann, J. I. TraPPE-UA Force Field for Acrylates and Monte Carlo Simulations for Their Mixtures with Alkanes and Alcohols. J. Phys. Chem. B 2009, 113 (18), 6415-6425. https://doi.org/10.1021/jp810558v.

(7) Vanommeslaeghe, K.; Hatcher, E.; Acharya, C.; Kundu, S.; Zhong, S.; Shim, J.; Darian, E.; Guvench, O.; Lopes, P.; Vorobyov, I.; et al. CHARMM General Force Field: A Force Field for Drug-like Molecules Compatible with the CHARMM All-Atom Additive Biological Force Fields. J. Comput. Chem. 2009, NA-NA. https://doi.org/10.1002/jcc.21367.

(8) Lopes, N. C.; Pascal, B. Molecular Force Field for Ionic Liquids Composed of Triflate or Bistriflylimide Anions. J. Chem. Phys. B 2004, 108 (1), 16893-16898. https://doi.org/10.1021/jp0476545. 
(9) Pesko, D. M.; Webb, M. A.; Jung, Y.; Zheng, Q.; Miller, T. F.; Coates, G. W.; Balsara, N. P. Universal Relationship between Conductivity and Solvation-Site Connectivity in Ether-Based Polymer Electrolytes. Macromolecules 2016, 49 (14), 5244-5255. https://doi.org/10.1021/acs.macromol.6b00851.

(10) Webb, M. A.; Savoie, B. M.; Wang, Z. G.; Miller, T. F. Chemically Specific Dynamic Bond Percolation Model for Ion Transport in Polymer Electrolytes. Macromolecules 2015, 48 (19), 7346-7358. https://doi.org/10.1021/acs.macromol.5b01437. 